\title{
Complejos estomáticos de veinticuatro taxa de Mimosa (Leguminosae) presentes en México
}

\section{Stomatal complexes of twenty-four species of Mimosa (Leguminosae) occurring in Mexico}

\author{
Susana Adriana Montaño-Arias ${ }^{1,2}$ (D), Sara Lucía Camargo-Ricalde,2 (D), Rosaura Grether' (D)
}

1 Universidad Autónoma Metropolitana-Iztapalapa, Departamento de Biología, División de Ciencias Biológicas y de la Salud, Apdo. postal 55-535, 09340 Cd. Mx., México.

2 Autoras para la correspondencia: arias_susan@hotmail.com;

slcr@xanum.uam.mx

Citar como:

Montaño-Arias, S. A., S. L. CamargoRicalde y R. Grether. 2018. Complejos estomáticos de veinticuatro taxa de Mimosa (Leguminosae) presentes en México. Acta Botanica Mexicana 122 97-107. DOl: http://dx.doi.org/10.21829/ abml22.2018.1260

Recibido: 27 de junio de 2017. Revisado: 24 de julio de 2017.

Aceptado: 21 de septiembre de 2017. Primero en línea: 3 de octubre de 2017.

Publicado: 1 de enero de 2018.

DOI:

http://dx.doi.org/10.21829/abml22. 2018.1260

\section{Resumen:}

Antecedentes y Objetivos: La clasificación infragenérica de Mimosa (Leguminosae) es compleja. La distinción entre los taxa se basa en caracteres morfológicos y anatómicos. Diversos autores mencionan que los complejos estomáticos son útiles en la taxonomía vegetal. En Mimosa, la anatomía foliar es un campo poco explorado, por lo que se desconocen los complejos estomáticos de más de $90 \%$ de sus taxa. Por lo anterior, el objetivo de este trabajo fue determinar, con fines taxonómicos, el tipo de complejo estomático de 24 taxa de Mimosa presentes en México.

Métodos: Se seleccionaron 24 taxa de Mimosa presentes en México. Por taxon, se eligieron tres individuos con altura y cobertura similar; se recolectó el mayor número de pinnas de las hojas; éstas se mezclaron para obtener una muestra compuesta por individuo. Se eligieron los folíolos de la parte media de cada pinna y se lavaron con agua destilada. Una vez aclarados, se deshidrataron y se elaboraron preparaciones permanentes con resina sintética.

Resultados clave: Este estudio abarcó tres de las cinco secciones de Mimosa. La sección Batocaulon fue la mejor representada, con la mayoría de los taxa pertenecientes a las series Acanthocarpae y Boreales. Se encontraron folíolos anfistomáticos en 22 de los taxa; mientras que los otros dos tienen folíolos hipostomáticos. Los 24 taxa estudiados presentan complejos estomáticos de tipo paracítico; aunque M. goldmanii, M. luisana, M. monancistra y M. pringlei var. pringlei, además de tener este tipo de complejos, muestran los de tipo anisocítico, excepto en M. benthamii var. benthamii que son anomocíticos. Conclusiones: Los taxa estudiados se agruparon considerando tres caracteres con valor taxonómico: $i$ ) folíolos hipostomáticos $v s$. folíolos anfistomáticos, $i i)$ presencia de un tipo de complejo estomático vs. dos tipos de complejos estomáticos, y iii) complejos estomáticos paracíticos y anisocíticos $v s$. complejos estomáticos paracíticos y anomocíticos.

Palabras clave: anatomía, estomas, Mimosa, morfología, taxonomía.

\section{ABSTRACT:}

Background and Aims: The infrageneric classification of Mimosa (Leguminosae) is complex. Distinction among taxa is based on morphological and anatomical characters. Various authors have stated that the stomatal complexes are valuable in plant taxonomy. In Mimosa, since leaf anatomy has been poorly explored, the stomatal complexes of over $90 \%$ of its taxa are still unknown. Therefore, the objective of this study was to determine, with taxonomic purposes, the stomatal complex type of 24 taxa of Mimosa occurring in Mexico.

Methods: Twenty-four taxa of Mimosa distributed in Mexico were selected. Per taxon, three individuals with similar height and coverage were chosen; a considerable amount of leaf pinnae was collected; pinnae were mixed in order to obtain a compound sample per taxon. Leaflets placed in the middle of the pinna were handpicked and washed with distilled water. Once the leaflets were cleared, they were dehydrated and permanent slides were prepared using synthetic resin.

Key results: The study included three of the five sections of Mimosa. Section Batocaulon was the best represented, where most of the taxa belong to series Acanthocarpae and Boreales. Amphistomatic leaflets were found in 22 of the taxa; while the other two have hypostomatic leaflets. The 24 studied taxa present paracytic stomatal complexes; though M. goldmanii, M. luisana, M. monancistra, and $M$. pringlei var. pringlei, additionaly show the anisocytic type, except in M. benthamii var. benthamii, which presents the anomocytic type.

Conclusions: The studied taxa were grouped considering three characters with taxonomic value: $i$ ) hypostomatic leaflets vs. amphistomatic leaflets, $i i)$ the presence of only one type of stomatal complex $v s$. the presence of two types of stomatal complexes, and iii) paracytic and anisocytic stomatal complexes $v s$. paracytic and anomocytic stomatal complexes.

Key words: anatomy, Mimosa, morphology, stomata, taxonomy. 


\section{INTRODUCCIÓN}

Tradicionalmente, la familia Leguminosae se ha dividido en tres subfamilias: Caesalpinioideae, Mimosoideae y Papilionoideae (Lewis et al., 2005). Una nueva clasificación de esta familia, basada en datos moleculares y morfológicos (LPWG, 2017) propone seis subfamilias: Duparquetioideae, Cercidoideae, Detarioideae, Dialioideae, Caesalpinoideae (incluyendo a la subfamilia Mimosoideae como sinónimo y formando un clado mimosoide anidado entre las tribus Caesalpinieae y Cassieae), y Papilionoideae; por lo que, actualmente, el género Mimosa L. podría ubicarse en la subfamilia Caesalpinoideae. Sin embargo, esta nueva clasificación no afecta la clasificación infragenérica de Mimosa ni los resultados de este trabajo.

En México, Mimosa cuenta con ca. 104 especies, de las cuales 54\% es endémico (Grether et al., 2015). De acuerdo con Barneby (1991) y Grether (1997) los taxa se distinguen, a nivel infragenérico, utilizando caracteres morfológicos como la forma de vida, tipo de inflorescencia, morfología de la flor (i.e. número de estambres; presencia o ausencia de estaminodios), hoja (i.e. número de pinnas, número de folíolos), fruto (i.e. valvas enteras o en artejos, número de semillas por fruto), semilla (i.e. color, tamaño del pleurograma) y aguijones (i.e. posición y número), entre otros. No obstante, la delimitación de secciones y series aún es compleja, por lo que se ha implementado la búsqueda de otros caracteres que aporten información adicional para el reconocimiento entre taxa.

Con esta visión, se han realizado estudios palinológicos (Caccavari, 1985, 1986, 1987; Chehaibar, 1988; Flores-Cruz et al., 2006) y de la anatomía de la madera (Marchiori, 1996; Montaño-Arias, 2010; Montaño-Arias et al., 2016, 2017). Sin embargo, dado el número de taxa comprendidos en el género Mimosa, los estudios con enfoque taxonómico se consideran escasos.

Igualmente, es relevante mencionar que los caracteres de las células epidérmicas, y los estomas y tricomas han sido usados en la taxonomía de las angiospermas (Stuessy, 1990); así como la anatomía de los complejos estomáticos (Rosati, 1991). En particular, el estudio anatómico de los complejos estomáticos comenzó en la década de 1960 con la observación de una tendencia de las células subsidiarias a formar patrones constantes en un mismo taxon (Fahn, 1967; Roth y Clausnitzer, 1969; Percy y Santa, 1976). Desde entonces, se han realizado estudios enfocados a la determinación de estos patrones en diferentes taxa.

El tipo de complejo estomático ha permitido diferenciar los taxa de las familias Smilacaceae (Baruah et al., 2012), Cactaceae (Eggli, 1984) y Gesneriaceae (RamírezRoa y Varela-Hernández, 2011), entre otras.

En el caso de las leguminosas, Percy y Santa (1976) reportaron el tipo de complejo estomático de 300 especies pertenecientes a diversos géneros como Inga Mill., Swartzia Schreb., Trifolium L., Hymenaea L., Canavalia Adans., Dioclea Kunth y Tamarindus L., entre otros. Asimismo, Araujo (2005) reporta que seis especies del género Erythrina L. han podido diferenciarse por la ubicación del complejo estomático presente.

Por su parte, Grosso et al. (1994) estudiaron el tipo de complejo estomático en 102 especies de Acacia Mill., reconociendo dos tipos de complejos, el anomocítico y el anisocítico. En el caso particular del género Mimosa, se conoce que sus taxa pueden presentar folíolos hipostomáticos o anfistomáticos, que la presencia de complejos estomáticos de tipo paracítico es común, pero pueden llegar a presentar más de un tipo de complejo (Bässler, 1985; Chiamolera et al., 2011; Begum y Borthakur, 2013; Rothe y Bathe, 2014). Sin embargo, la anatomía foliar es un campo poco explorado. Con base en lo anterior, el objetivo de este estudio fue determinar, con fines taxonómicos, el tipo de complejo estomático de 24 taxa del género Mimosa (Leguminosae) presentes en México, bajo el supuesto de que, al aumentar el número de taxa analizados, habrá variación del tipo de complejos estomáticos, lo que permitirá la distinción de los taxa.

\section{Material y Métodos}

\section{Material vegetal}

Se seleccionaron 24 taxa de Mimosa presentes en México, los cuales presentaron forma de vida arbórea (un solo 
tronco leñoso, con ramificación a partir del metro de altura) o arbustiva (al menos dos troncos leñosos, con ramificación desde la base); dichos taxa pertenecen a diferentes secciones y series del género (Cuadro 1).

\section{Selección y tratamiento de los folíolos}

Considerando la variabilidad de cada taxon, se seleccionaron tres individuos de cada uno. La copa de cada individuo se dividió en cuatro cuadrantes para la selección de pinnas de las hojas. De cada cuadrante, se recolectó el mayor nú- mero de pinnas; éstas se mezclaron para obtener una muestra compuesta por individuo. Las pinnas se depositaron en frascos, se fijaron con FAA (1:1:1) y se mantuvieron a temperatura ambiente en el laboratorio de Biosistemática de Leguminosas, UAM Iztapalapa, en la Ciudad de México. Los ejemplares de referencia están depositados en el Herbario Metropolitano (UAMIZ), de la Universidad Autónoma Metropolitana, Unidad Iztapalapa.

En el laboratorio, se eligieron los folíolos de la parte media de cada pinna y se lavaron con agua destilada.

Cuadro 1: Clasificación de los taxa de Mimosa L. (Leguminosae) estudiados según Barneby (1991) y Grether (2000).

\begin{tabular}{|c|c|c|c|c|}
\hline Sección & Serie & Subserie & Especie & Variedad \\
\hline \multirow[t]{16}{*}{ Batocaulon } & Acanthocarpae & & M. aculeaticarpa Ortega & aculeaticarpa \\
\hline & & & M. biuncifera Benth. & \\
\hline & & & M. galeottii Benth. & \\
\hline & & & M. grahamii A. Gray & grahamii \\
\hline & & & M. lacerata Rose & \\
\hline & & & M. purpusii Brandegee & \\
\hline & Distachyae & & M. benthamii J.F. Macbr. & benthamii \\
\hline & & & M. luisana Brandegee & \\
\hline & & & M. polyantha Benth. & \\
\hline & Boreales & & M. calcicola B.L. Rob. & \\
\hline & & & M. depauperata Benth. & \\
\hline & & & M. monancistra Benth. & \\
\hline & & & M. pringlei S. Watson & pringlei \\
\hline & & & M. similis Britton \& Rose & \\
\hline & & & M. texana (A. Gray) Small & filipes (Britton \& Rose) Barneby \\
\hline & & & M. texana (A. Gray) Small & texana \\
\hline Habbasia & Habbasia & & M. pigra $\mathrm{L}$. & pigra \\
\hline \multirow[t]{7}{*}{ Mimosa } & Mimosa & Mimosa & M. albida Humb. \& Bonpl. ex Willd. & albida \\
\hline & & & M. albida Humb. \& Bonpl. ex Willd. & strigosa (Willd.) B.L. Rob. \\
\hline & Lactifluae & & M. caerulea Rose & \\
\hline & & & M. goldmanii B.L. Rob. & \\
\hline & & & M. lactiflua Delile ex Benth. & \\
\hline & & & M. tricephala Schltdl. \& Cham. & \\
\hline & & & Mimosa sp. & \\
\hline
\end{tabular}


Posteriormente, se colocaron en cloro comercial durante 4 a $72 \mathrm{~h}$ a temperatura ambiente (Sandoval, 2005). Una vez aclarados, se deshidrataron en alcoholes graduales de 50 a $100 \%$, y se elaboraron preparaciones permanentes con resina sintética (10 preparaciones/taxon). Por preparación, se observaron 20 folíolos, haciendo un total de 200 folíolos por taxon. De los 24 taxa, se observaron 4800 folíolos en total. Se utilizó un microscopio óptico (Zeiss, Primo-Star, Alemania). Las preparaciones permanentes están bajo resguardo del laboratorio de Biosistemática de Leguminosas.

\section{Resultados}

La sección Batocaulon fue la mejor representada; en ella, los taxa estudiados están ubicados principalmente en las series Acanthocarpae y Boreales (Cuadro 1).

Los folíolos anfistomáticos se encontraron en 22 de los taxa. En el caso de Mimosa benthamii J.F. Macbr. var. benthamii y M. caerulea Rose, las hojas son de tipo hipostomático. Asimismo, los 24 taxa estudiados presentan complejos estomáticos de tipo paracítico, caracterizados por presentar dos células acompañantes paralelas al eje longitudinal de las células oclusivas (Cuadro 2, Fig. 1).

En el caso de M. goldmanii B.L. Rob., M. luisana Brandegee, M. monancistra Benth., M. pringlei var. pringlei $\mathrm{S}$. Watson, M. similis Britton \& Rose y M. tricephala Schltdl. \& Cham., además de presentar complejos estomáticos de tipo paracítico, tienen complejos anisocíticos, los que se caracterizan por tener tres células acompañantes de distintos tamaños que rodean a las células oclusivas. Cabe señalar que éstos se encuentran en menor proporción, pero en la misma superficie epidérmica (Fig. 2). Mimosa benthamii var. benthamii también tiene complejos estomáticos paracíticos y presenta otros anomocíticos.

De manera adicional, se observó que los folíolos de M. benthamii var. benthamii, M. calcicola B.L. Rob., M. luisana, M. purpusii Brandegee y M. tricephala presentan abundantes tricomas unicelulares. En el caso de $M$. purpusii y M. tricephala, la presencia de dichos tricomas dificultó la observación de sus complejos estomáticos.

\section{DISCUSIÓN}

Los resultados obtenidos permiten separar a los taxa de Mimosa estudiados en dos grupos: i) los que presentan folíolos hipostomáticos, y ii) los que presentan folíolos anfistomáticos.

Mimosa benthamii var. benthamii y M. caerulea son los únicos taxa que tienen folíolos hipostomáticos. Asimismo, Bässler (1985) observó este tipo de folíolos en M. fagaracantha Griseb., M. pulverulenta Urb., M. ekmanii Urb. y M. apleura Urb. Chiamolera et al. (2011) también los observaron en M. scabrella Benth. Por otra parte, los 22 taxa restantes presentan folíolos de tipo anfistomático, al igual que $M$. distachya Cav., M. berlandieri A. Gray (=M. pigra L. var. berlandieri (A. Gray ex Torr.) B.L. Turner), M. invisa Mart. (=M. diplotricha C. Wright ex Sauvalle var. diplotricha), M. viva Vell. (Bässler, 1985), M. diplotricha var. diplotricha, M. himalayana Gamble y M. pudica L. (Begum y Borthakur, 2013). Por lo expuesto, son 29 taxa con esta característica, de las ca. 530 especies conocidas a nivel mundial (Särkinen et al., 2011; Simon et al., 2011). En forma adicional, Percy y Santa (1976) señalan el tipo de complejo estomático paracítico, presente en M. colombiana Britton \& Killip y M. sensitiva L. Sin embargo, no hacen referencia al tipo de folíolo presente (hipostomático o anfistomático), al igual que Chiamolera et al. (2011), quienes no lo indican en M. scabrella. En la actualidad, se contabilizan ca. 38 taxa estudiados, lo que representan ca. 7\% de los taxa de Mimosa a nivel mundial, siendo todavía prematuro el afirmar que el tipo de complejo estomático y su presencia en una o en ambas superficies del folíolo pudiese ser considerado un carácter con valor taxonómico a nivel genérico o infragenérico.

De la misma forma, los taxa de Mimosa estudiados presentan complejos estomáticos de tipo paracítico, los que fueron considerados por Metcalfe y Chalk (1979) como distintivos de las leguminosas. Esto fue confirmado por Percy y Santa (1976) al observar el mismo tipo de complejo estomático en 300 especies de esta familia.

Estudios posteriores reportan la presencia del complejo estomático paracítico en folíolos de taxa pertene- 

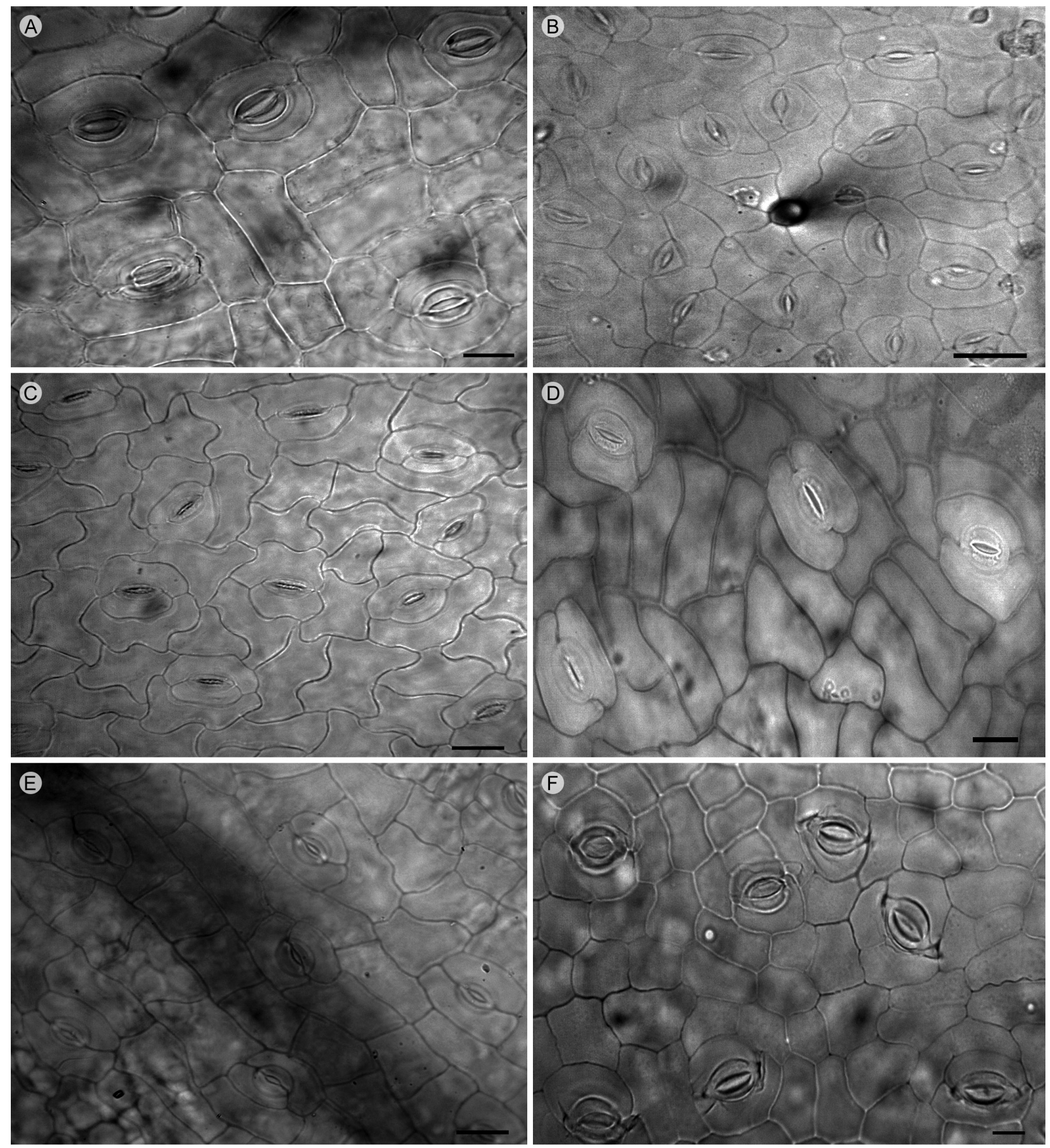

Figura 1: Complejos estomáticos paracíticos. A. Mimosa aculeaticarpa Ortega var. aculeaticarpa; B. M. albida Humb. \& Bonpl. ex Willd. var. strigosa (Willd.) B.L. Rob.; C. M. caerulea Rose; D. M. lactiflua Delile ex Benth.; E. M. polyantha Benth.; F. M. texana (A. Gray) Small var. texana. Escala: $20 \mu \mathrm{m}$. 


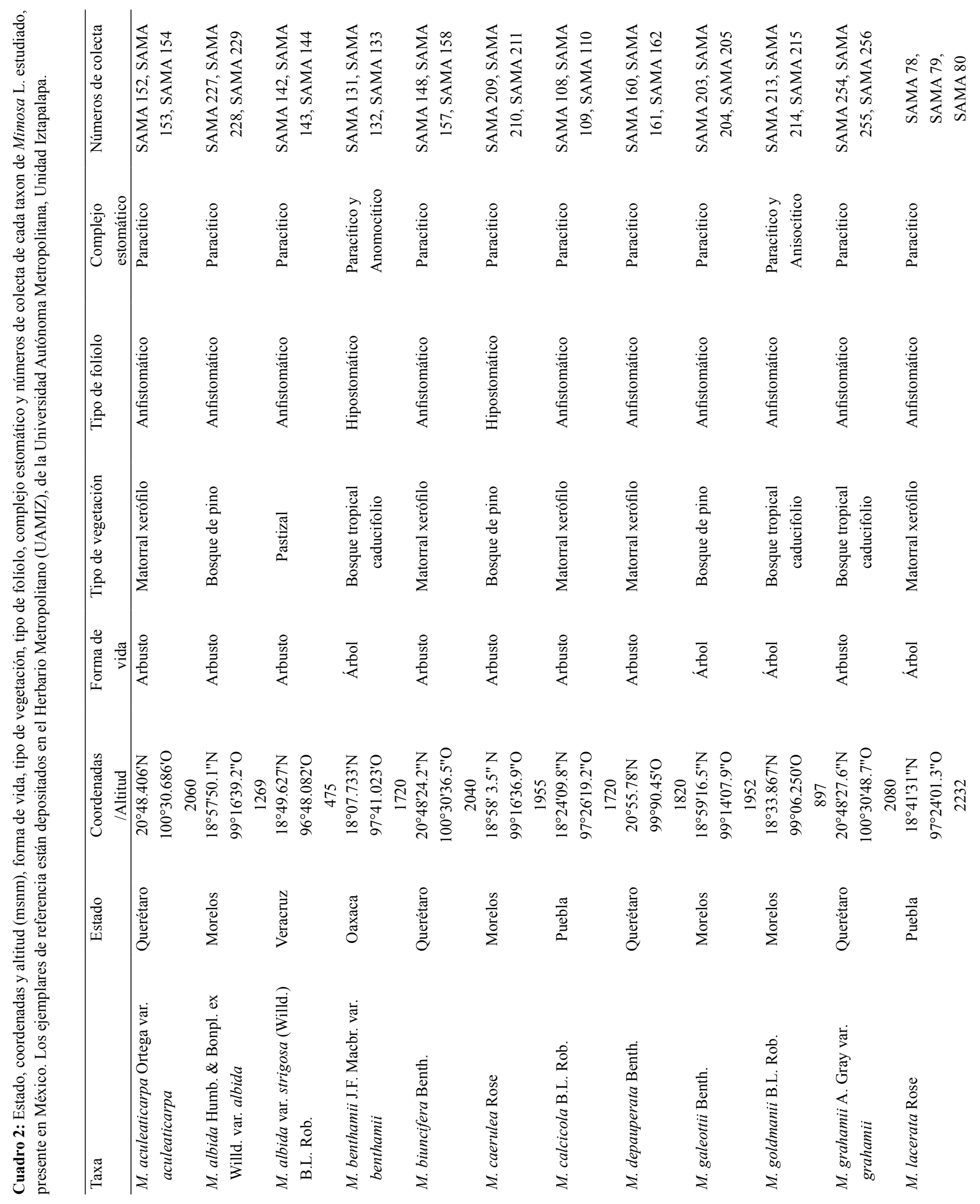




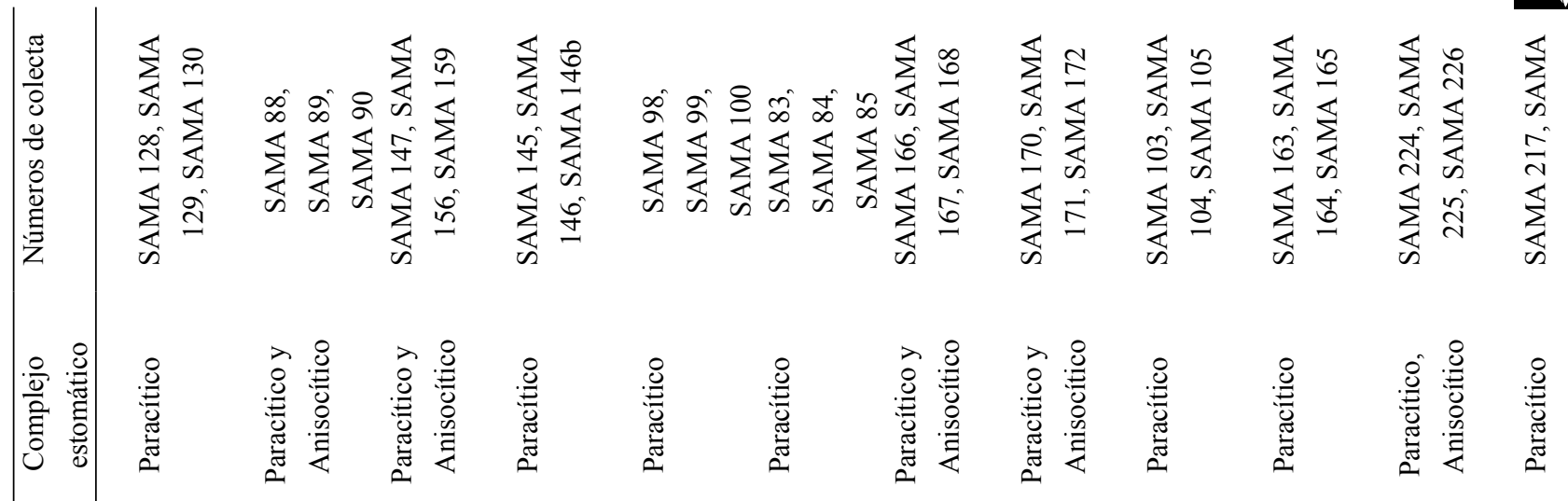
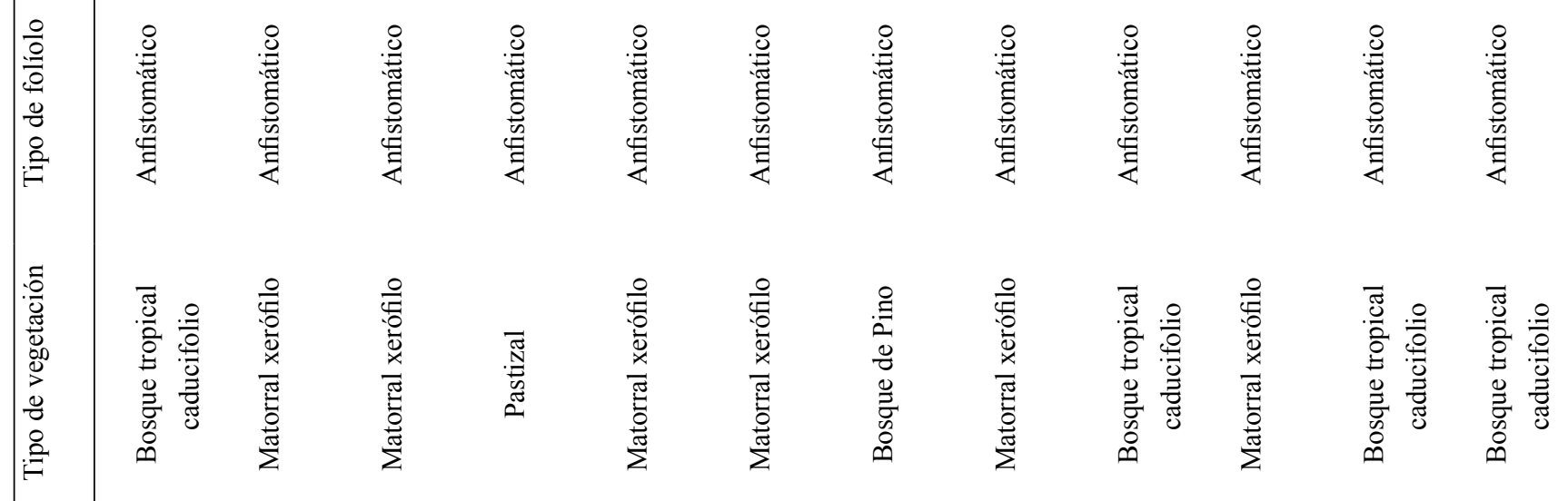

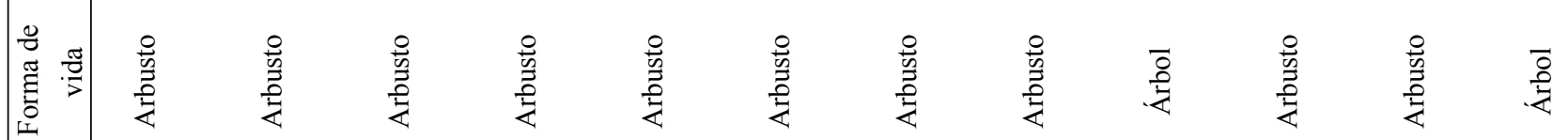

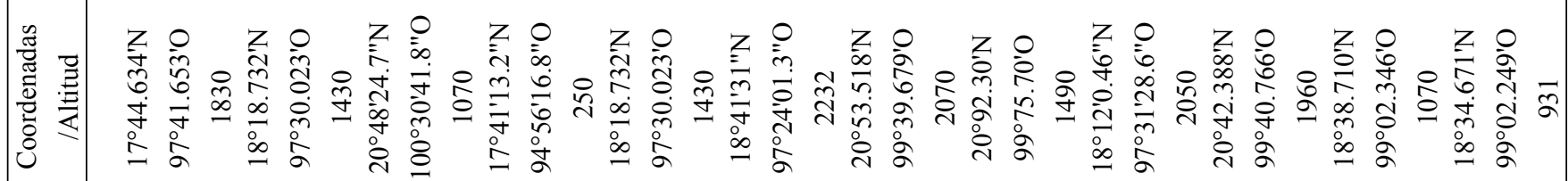

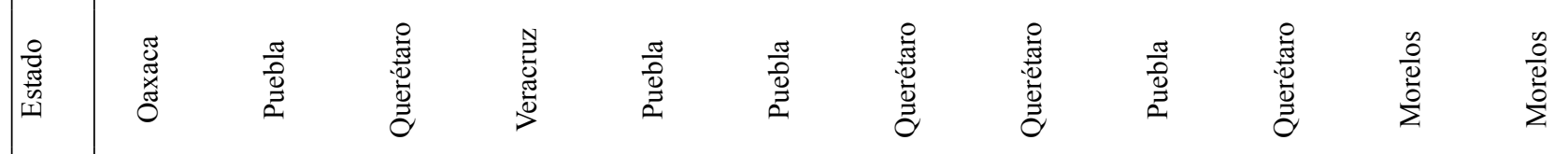

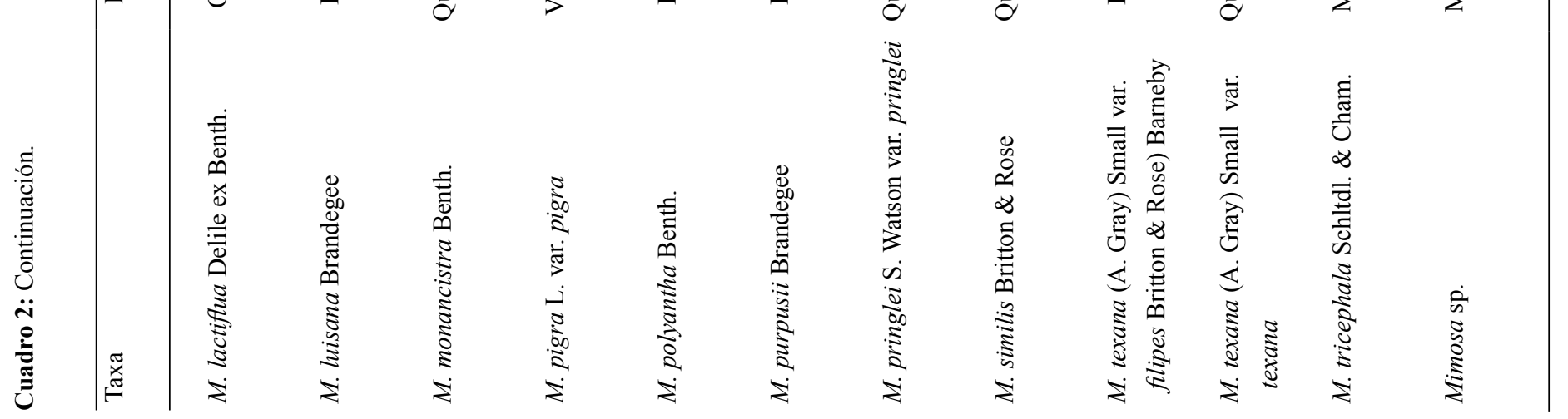



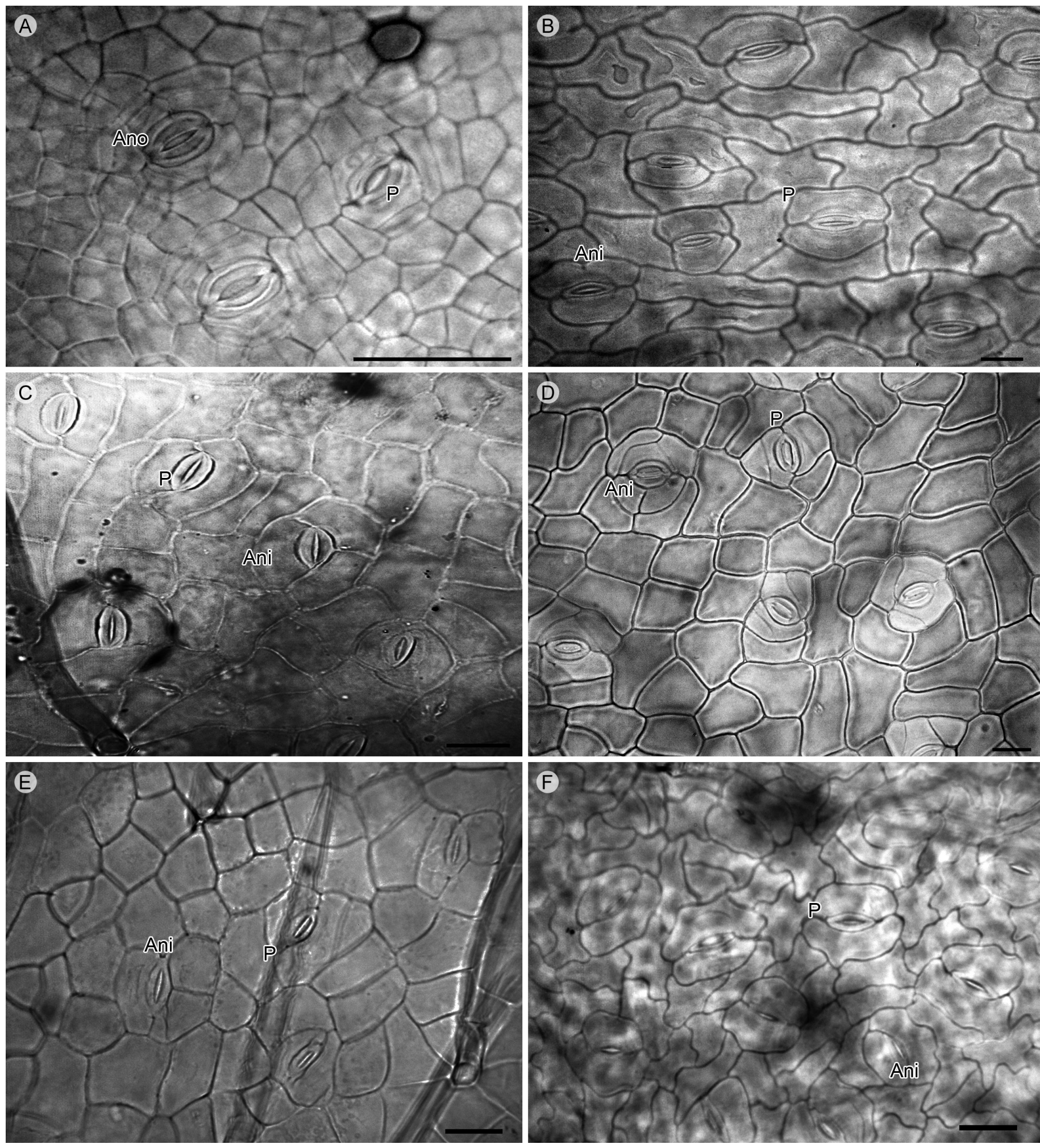

Figura 2: Complejos estomáticos anomocíticos (Ano), anisocíticos (Ani) y paracíticos (P). A. Mimosa benthamii J.F. Macbr. var. benthamii; B. M. goldmanii B.L. Rob.; C. M. monancistra Benth.; D. M. pringlei S. Watson var. pringlei; E. M. similis Britton \& Rose; F. M. tricephala Schltdl. \& Cham. Escala: $20 \mu \mathrm{m}$ 
cientes a dos géneros de mimosoideas: Acacia (Duarte y Wolf, 2005; Hernández y Arambarri, 2010) y Prosopis L. (Vilela, 1993); así como de los géneros Astragalus L. (Fahmy, 1997) e Indigofera L. (Martínez, 1997), pertenecientes a la subfamilia Papilionoideae.

En el caso de Mimosa, los complejos estomáticos paracíticos ya habían sido observados en M. albida Humb. \& Bonpl. ex Willd., M. colombiana, M. pigra L., M. pudica, M. pulverulenta y M. sensitiva (Shah et al., 1972; Percy y Santa, 1976; Bässler, 1985; Rothe y Bathe, 2014). Sin embargo, no se especifica la variedad estudiada en estas especies, con excepción de M. colombiana y M. pulverulenta, ya que no se reconoce ninguna variedad (Barneby, 1991).

Por otra parte, Begum y Borthakur (2013) reportaron complejos estomáticos paracíticos en $M$. diplotricha var. diplotricha, M. himalayana y M. pudica; aunque señalan que $M$. himalayana, además del complejo estomático paracítico, presenta complejos anisocíticos. Stenglein et al. (2005) consideran que la presencia de más de un tipo de complejo estomático es común en leguminosas. Sin embargo, hasta ahora era distintivo de $M$. himalayana, pero en este estudio también se observó en $M$. benthamii var. benthamii, M. goldmanii, M. luisana, M. monancistra, $M$. pringlei var. pringlei y $M$. similis.

Por lo tanto, la presencia de dos tipos de complejos estomáticos en los folíolos de un mismo taxon permite separarlos en dos grupos: $i$ ) taxa con un sólo tipo de complejo estomático, y ii) taxa con dos tipos de complejo estomático, lo que permite distinguir por la combinación de tipos de complejos estomáticos presentes. Por ejemplo, $M$. benthamii var. benthamii es el único taxon con estomas paracíticos y anomocíticos; mientras que $M$. goldmanii, M. luisana, M. monancistra, M. pringlei y $M$. similis presentan complejos estomáticos paracíticos y anisocíticos; por lo que, a nivel específico, la presencia de dos tipos de complejos estomáticos en un mismo folíolo puede considerarse como un carácter útil para diferenciar entre los taxa. Sin embargo, la presencia de complejos estomáticos paracíticos, o de dos tipos de complejos en la misma superficie foliar, no es exclusivo de una serie.
Asimismo, a nivel de las secciones del género, es prematuro considerarlo un carácter útil, ya que, aunque se tienen representadas todas las secciones de Mimosa, únicamente se han estudiado los estomas de un taxon de las secciones Calothamnos, Habbasia y Mimadenia.

\section{CONCLUSIONES}

Los taxa estudiados se agruparon considerando tres caracteres con valor taxonómico: $i$ ) folíolos hipostomáticos $v s$. folíolos anfistomáticos, ii) presencia de un tipo de complejo estomático vs. dos tipos de complejos estomáticos, y iii) complejos estomáticos paracíticos y anisocíticos vs. complejos estomáticos paracíticos y anomocíticos.

Hasta este momento, se conoce el tipo de complejo estomático de ca. 7\% de los taxa de Mimosa a nivel mundial y el $23 \%$ de los taxa presentes en México, por lo que todavía se requiere estudiar el 93 y $77 \%$ de los taxa de Mimosa presentes en el mundo y en México, respectivamente.

\section{CONTRIBUCIONES DE AUTORES}

SAMA concibió y diseñó el estudio, trabajo de campo y desarrollo experimental. SAMA, SLCR y RG realizaron el análisis e interpretación de los datos. SAMA redactó el manuscrito con ayuda de SLCR y RG. Todos los autores contribuyeron con la discusión, revisión y aprobación del manuscrito final.

\section{FINANCIAMIENTO}

Este estudio fue apoyado por la Universidad Autónoma Metropolitana, Unidad Iztapalapa, proyecto "Biología y ecología del género Mimosa (Leguminosae-Mimosoideae) en ecosistemas áridos y semiáridos de México: el caso del Valle de Tehuacán-Cuicatlán como un sistema modelo. Fase 1".

\section{Literatura Citada}

Araujo, A. V. 2005. Estudio taxonómico e histológico de seis especies del género Erythrina L. (Fabaceae). Tesis de maestría. Facultad de Ciencias Biológicas. Lima, Perú. $123 \mathrm{pp}$. 
Barneby, R. C. 1991. Sensitivae Censitae. A description of the genus Mimosa L. (Mimosaceae) in the New World. Memoirs of The New York Botanical Garden 65: 1-835.

Baruah, S., S. K. Borthakur y A. Ahmed. 2012. Taxonomic status of certain members of Smilax Linnaeus (Smilacaceae) based on their foliar epidermal structures. Pleione 6(1): 87-92.

Bässler, M. 1985. Die Gattung Mimosa L. (Leguminosae-Mimosoideae) in Cuba. Feddes Repertorium 96(7-10): 581-611.

Begum, A. y S. K. Borthakur. 2013. Comparative micromorphological studies on leaf epidermis in three species of Mimosa Linnaeus (Mimosaceae) from Assam, India. Pleione 7(1): 1-7.

Caccavari, M. A. 1985. Granos de polen de las Leguminosas de la Argentina IV. Género Mimosa. Boletín de la Sociedad Argentina de Botánica 24(1-2): 151-167.

Caccavari, M. A. 1986. Estudio de los caracteres del polen de las Mimosa-Lepidotae. Pollen et Spores 28(1): 29-42.

Caccavari, M. A. 1987. Estudio de los caracteres del polen de Mimosa-Glanduliferae. VII Simposio Argentino de Paleobotánica y Palinología, Actas: 141-144.

Chehaibar, M. T. 1988. Estudio taxonómico de la serie Xantiae y especies afines del género Mimosa (Leguminosae). Tesis de maestría en Ciencias. Facultad de Ciencias, Universidad Nacional Autónoma de México. México, D.F., México. 107 pp.

Chiamolera, L. D. B., A. C. Ângelo, M. R. Boeger y F. C. Araújo. 2011. Aspectos anatômicos e fisiológicos de folhas de Mimosa scabrella de áreas com diferentes graus de sucessão. Floresta 41(3): 435-450. DOI: http://dx.doi. org/10.5380/rf.v41i3.23990

Duarte, M. R. y S. Wolf. 2005. Anatomical characters of the phyllode and stem of Acacia podalyriifolia A. Cunn. ex G. Don (Fabaceae). Revista Brasileira de Farmacognosia 15(1): 71-76. DOI: http://dx.doi.org/10.1590/S0102$695 \times 2005000100015$

Eggli, U. 1984. Stomatal types of Cactaceae. Plant Systematics and Evolution 146(3-4): 197-214. DOI: https://doi. org/10.1007/BF00989546

Fahmy, G. M. 1997. Leaf anatomy and its relation to the ecophysiology of some non-succulent desert plants from
Egypt. Journal of Arid Environments 36(3): 499-525. DOI: https://doi.org/10.1006/jare.1996.0217

Fahn, A. 1967. Plant anatomy. Pergamon Press. Oxford, UK. $534 \mathrm{pp}$.

Flores-Cruz, M. F., H. D. S. Lira, A. Martínez-Bernal y M. E. Fraile. 2006. Morfología del polen de Mimosa serie Quadrivalves (Leguminosae, Mimosoideae). Acta Botanica Mexicana 77: 1-13. DOI: http://dx.doi. org/10.21829/abm77.2006.1022

Grether, R. 1997. Revisión taxonómica del género Mimosa (Leguminosae) en Mesoamérica. Tesis de doctorado. Facultad de Ciencias, Universidad Nacional Autónoma de México. México, D.F., México. 370 pp.

Grether, R. 2000. Nomenclatural changes in the genus Mimosa (Fabaceae, Mimosoideae) in Southern Mexico and Central America. Novon 10(1): 29-37. DOI: https://doi. org $/ 10.2307 / 3393180$

Grether, R., S. L. Camargo-Ricalde, A. Martínez-Bernal, S. Montaño-Arias y M. E. Fraile 2015. Diversity and geographical distribution patterns of the genus Mimosa (Mimosoideae) in the United States, Mexico, and Central America. In: Fortunato, E. H. (ed.). V Conferencia Internacional de Leguminosas (VILC). Fundación Centro de Integración Comunicación, Cultura y Salud (CICCUS). Buenos Aires, Argentina. 224 pp.

Grosso, B., M. Saint-Martin y J. Vassal. 1994. Stomatal types of the genus Acacia (Fabaceae, Mimosoideae): an appraisal of diversity and taxonomic interest. Botanical Journal of the Linnean Society 116(4): 325-341. DOI: http://dx.doi. org/10.1111/j.1095-8339.1994.tb00437.x

Hernández, M. P. y A. M. Arambarri. 2010. Stomatal distribution, stomatal density and daily leaf movement in Acacia aroma (Leguminosae). Boletín de la Sociedad Argentina de Botánica 45: 273-284.

Lewis, G. P., B. Schrire, B. Mackinder y M. Lock (eds.). 2005. Legumes of the World. Royal Botanic Gardens, Kew. Kew, UK. 592 pp.

LPWG. 2017. A new subfamily classification of the Leguminosae based on a taxonomically comprehensive phylogeny. Taxon 66(1): 44-77. DOI: https://doi. org/10.12705/661.3 
Marchiori, J. N. C. 1996. Anatomia do xilema secundário de Mimosa incana (Spreng.) Benth. Ciência Florestal 6(1): 53-63.

Martínez, Q. E. 1997. Taxonomic significance of foliar dermotypes and floral trichomes in some Cuban taxa of Indigofera L. (Fabaceae-Faboideae). Polibotánica 6: 1-18.

Metcalfe, C. R. y L. Chalk. 1979. Anatomy of the Dicotyledons, Vol. I. Clarendon Press, Oxford. London, UK. 276 pp.

Montaño-Arias, S. A. 2010. Anatomía comparada de la madera de especies arbóreas mexicanas del género Mimosa Sección Batocaulon (Leguminosae). Tesis de maestría. Universidad Autónoma Metropolitana, Unidad Iztapalapa. México, D.F., México. 141 pp.

Montaño-Arias, S. A., S. L. Camargo-Ricalde y R. Grether. 2016. Anatomía de la madera de tres especies de Mimosa (Leguminosae-Mimosoideae) distribuidas en México. Madera y Bosques 22(1): 191-202. DOI: http://dx.doi. org/10.21829/myb.2016.221486

Montaño-Arias, S. A., S. L. Camargo-Ricalde, R. Grether y D. Díaz-Pontones. 2017. Ecoanatomía de la madera de dos taxa mexicanos del género Mimosa (LeguminosaeMimosoideae). Acta Botanica Mexicana 118: 105-120. DOI: http://dx.doi.org/10.21829/abm118.2017.1203

Percy, D. y J. Santa. 1976. Patrón estomático paracítico en Leguminosas. I. Actualidades Biológicas 5: 80-87.

Ramírez-Roa, A. y G. Varela-Hernández. 2011. Características anatómicas de hoja y flor con importancia taxonómica para la delimitación de cuatro especies en el género Moussonia (Gesneriaceae). Annals of the Missouri Botanical Garden 98: 413-429. DOI: https://doi.org/10.3417/2005150

Rosati, V. R. 1991. Caracteres epidérmicos foliares de valor diagnóstico en la identificación de plantas leñosas del Chaco occidental. AgriScientia 8: 41-53

Roth I. e I. Clausnitzer. 1969. Los tipos de desarrollo estomático en las angiospermas. Acta Botanica Venezuelica 4(1/4): 259-292.
Rothe, S. P. y A. U. Bathe. 2014. Comparative leaf anatomy of sensitive plants found in Akola District. International Journal of Pharmaceutical and Chemical Sciences 3(2): 496-500.

Sandoval, E. 2005.Técnicas aplicadas al estudio de la anatomía vegetal. Cuadernos 38. Universidad Nacional Autónoma de México, Instituto de Biología. México, D.F., México. $278 \mathrm{pp}$.

Särkinen, T. E., J. L. Marcelo-Peña, A. D. Yomona, M. F. Simon, R. T. Pennington y C. E. Hughes. 2011. Underestimated endemic species diversity in the dry inter-Andean valley of the Río Marañón, northern Peru: An example from Mimosa (Leguminosae, Mimosoideae). Taxon 60(1): 139-150.

Shah, G. L., M. H. Parabia, M. J. Kothari. 1972. Epidermal structures and stomatal ontogeny in some Mimosaceae. Annals of Botany 36(4): 823-835. DOI: https://doi. org/10.1093/oxfordjournals.aob.a084638

Simon, M. F., R. Grether, L. P. de Queiroz, T. E. Särkinen, V. F. Dutra y C. E. Hughes 2011. The evolutionary history of Mimosa (Leguminosae): towards a phylogeny of the sensitive plants. American Journal of Botany 98(7): 12011221. DOI: http://dx.doi.org/10.3732/ajb.1000520

Stenglein, S. A., A. M. Arambarri, M. C. Menendez-Sevillano y P. A. Balatti. 2005. Leaf epidermal characters related with plant's passive resistance to pathogens vary among accessions of wild beans Phaseolus vulgaris var. aborigineus (Fabaceae-Phaseoleae). Flora 200(3): 285295. DOI: https://doi.org/10.1016/j.flora.2005.01.004

Stuessy, T. F. 1990. Plant taxonomy, the systematic evaluation of comparative data. Columbia University Press. New York, USA. 514 pp.

Vilela, A. E. 1993. Anatomia foliar de Prosopis (LeguminosaeMimosoideae): estrategias adaptativas a diferentes ambientes en Prosopis nigra. Darwiniana 32(1-4): 99107. 